safely requires a dedicated service as outlined in BSG guidelines for IBD, and not necessarily a tertiary care referral!

Disclosure of Interest None Declared.

\section{PWE-106 SMALL INTESTINE ULTRASONOGRAPHY WITH ORAL CONTRAST (SICUS) FOR THE DETECTION OF SMALL BOWEL COMPLICATIONS IN CROHN'S DISEASE: CORRELATION WITH INTRA-OPERATIVE FINDINGS}

${ }^{1} \mathrm{~S}$ Kumar*, ${ }^{1} \mathrm{~A}$ Hakim, ${ }^{1} \mathrm{C}$ Alexakis, ${ }^{1} \mathrm{~V}$ Chhaya, ${ }^{2} \mathrm{D}$ Tzias, ${ }^{2} \mathrm{~J}$ Pilcher, ${ }^{1} \mathrm{R}$ Pollok. ${ }^{1}$ Department of Gastroenterology, St George's Hospital, London, UK; ' ${ }^{2}$ epeartment of Radiology, St George's Hospital, London, UK

\subsection{6/gutjnl-2014-307263.366}

Introduction SICUS accurately assesses small bowel lesions in patients with Crohn's disease (CD) without exposure to diagnostic medical radiation. ${ }^{1}$ Its role in identifying intra-abdominal complications associated with CD remains to be confirmed. The aim of this retrospective study was to compare the diagnostic sensitivity of SICUS with subsequent surgical findings.

Methods Patients with CD evaluated by SICUS who subsequently required bowel resection within 6 months were identified. Radiological findings and operation notes were collated. The accuracy and agreement of SICUS to detect the site and length of strictures, fistulae, abscesses and mucosal thickening, was compared with surgical findings and assessed by kappa $(\kappa)$ coefficient statistical analysis. Stricture lengths were compared using student's t-test. CRP and platelet count were recorded within 2 weeks of SICUS and surgery as surrogate markers of disease activity.

Results A total of 25 patients (12 male) with a mean age of 29.9 years were included in the study. Mean time from SICUS to respective bowel surgery was 91.5 days (Range 5-176). Ultrasonographic and surgical inter-rater agreement was good for the presence of strictures $(\kappa=0.73$, sensitivity and specificity both $88 \%)$, their number $(\kappa=0.65,95 \% \mathrm{CI}: 0.31-0.96)$ and stricture site $(\kappa=0.72,95 \%$ CI: $0.44-1.00)$. Stricture length was $7.4 \pm$ $1.5 \mathrm{~cm}$ identified at surgery vs. $5.8 \pm 1.8 \mathrm{~cm}$ by SICUS (NS). Agreement was excellent for the presence of fistulae $(\kappa=0.82$, sensitivity $86 \%$, specificity $94 \%)$, location of fistula $(\kappa=0.92$, 95\% CI: $0.76-1.00)$, presence of abscess $(\kappa=0.87$, sensitivity $100 \%$, specificity $95 \%)$ and its location $(\kappa=0.87,95 \% \mathrm{CI}$ : $0.63-1.00)$. Agreement was moderate for mucosal wall thickening $(\kappa=0.51$, sensitivity 95\%, specificity 50\%). Markers of inflammation (CRP and platelet count) showed no significant difference at the time of SICUS and surgery.

Conclusion SICUS accurately identifies small bowel complications in $\mathrm{CD}$ and offers an alternative in the pre-operative stage of CD complications. Its wider use should be adopted.

\section{REFERENCE}

1 Chatu S, Pilcher J, Saxena SK, et al. Diagnostic accuracy of small intestine ultrasonography using an oral contrast agent in Crohn's disease: comparative study from the UK. Clin Radiol 2012 Jun;67(6):553-9

Disclosure of Interest None Declared.

\section{PWE-107 ACCURACY OF MAGNETIC RESONANCE ENTEROGRAPGY IN PREDICTING ANASTOMOTIC STENOSIS IN RECURRENT CROHN'S DISEASE}

${ }^{1} S S$ Poon*, ${ }^{2} \mathrm{R}$ Wiles, ${ }^{2} \mathrm{~F}$ Ammad, ${ }^{2} \mathrm{P}$ Healey, ${ }^{3} \mathrm{~S}$ Subramanian. ${ }^{1} \mathrm{Faculty}$ of Medicine, University of Liverpool, UK; ${ }^{2}$ Department of Radiology, Royal Liverpool and Broadgreen University Hospital, Liverpool, UK; ${ }^{3}$ Department of Gastroenterology, Royal Liverpool and Broadgreen University Hospital, Liverpool, UK

10.1136/gutjn-2014-307263.367

\begin{tabular}{lll} 
Abstract PWE-107 Table 1 & Baseline characteristics & \\
\hline & Median & Range \\
\hline Age (years) & 44 & $29-71$ \\
Disease duration (years) & 23.5 & $5-45$ \\
Time since last resection (years) & 7 & $4-25$ \\
Time between MRE and endoscopy (days) & 46 & $3-144$ \\
\hline
\end{tabular}

Introduction Up to $80 \%$ of patient with Crohn's disease (CD) undergo intestinal resection, commonly an ileocaecal resection. Eighty percent of patients develop endoscopic recurrence at the anastamotic site at 1 year and 50\% develop clinical recurrence at 3 years. The severity of endoscopic recurrence varies from no endoscopic lesions to anastamotic stenosis and/or diffuse inflammation. Direct endoscopic visualisation is recommended to detect recurrence but it is invasive. Magnetic resonance enterography (MRE) has become a standard imaging investigation for CD but only few studies have evaluated its utility in recurrent anastamotic stenosis. Accurate characterisation of recurrence grade is critical as strictures up to $5 \mathrm{cms}$ can be successfully treated with endoscopic balloon dilatation. We evaluated the utility of MRE in the assessment of anastamotic stenosis in recurrent $\mathrm{CD}$.

Methods This retrospective study was done at the Royal Liverpool Hospital and included all CD patients who underwent endoscopic balloon dilatation for anastomotic stenosis between 2009-2013. Patients who had an MRE done within 6 months of the endsocopic procedure were eligible for inclusion. MRE was done following administration of oral polyethylene glycol solution and sequences were analysed for the presence of stenosis, length of stenosis, pre-stenotic dilation and the presence of enhancement by an experienced gastrointestinal radiologist. The length and presence of stenosis was extracted from the endoscopy report and correlated against the MRE findings.

Results 16 patients were included in the study $(5$ male, 11 female). There was good agreement between endoscopy and MRE for the presence of anastamotic stenosis. Using endoscopy as the gold standard, the sensitivity and specificity of MRE in detecting anastomotic stenosis was $86 \%$ and $100 \%$ respectively. A significant variation was noted in the length of stenosis as assessed by MRE $(45 \pm 12 \mathrm{~mm}$, mean $\pm \mathrm{SE})$ and endoscopy $(20$ $\pm 3 \mathrm{~mm}$, mean $\pm \mathrm{SE}$ ), two sided $\mathrm{p}<0.05$. All patients underwent successful endoscopic dilatation.

Conclusion MRE is an accurate tool for predicting the presence of anastomotic stenosis in recurrent CD. However, the discrepancy observed in the length of stenosis between the two modalities may mean suitable patients for endoscopic dilatation are missed. Therefore, endoscopy and MRE should be used as complementary tools in the assessment of anastamotic stenosis. Disclosure of Interest None Declared.

\section{PWE-108 FACTORS INFLUENCING THE MANAGEMENT OF INFLAMMATORY BOWEL DISEASE IN PRIMARY CARE}

${ }^{1} \mathrm{SA}$ James*, ${ }^{2} \mathrm{CR}$ May, ${ }^{2} \mathrm{M}$ Simon, ${ }^{1} \mathrm{JF}$ Cummings. ${ }^{1}$ Department of Gastroenterology, University Hospital Southampton NHS Foundation Trust, UK; ${ }^{2}$ Faculty of Health Sciences, University of Southampton, Southampton, UK

\subsection{6/gutjnl-2014-307263.368}

Introduction Between 30-70\% of United Kingdom (UK) adults with Inflammatory Bowel Disease (IBD) are managed solely by 
their General Practitioner (GP). ${ }^{1,2}$ There is, however, limited knowledge as to how UK GPs manage this patient group and whether GP-led care meets IBD Standards. ${ }^{3}$ This study aimed to identify factors influencing long-term follow-up of adults with IBD by GPs; achieved through examining GPs' knowledge and management of IBD, including exploration of future IBD-care models.

Methods A non-probability, convenience sample of 34 Senior Partner GPs and 130 Colleague GPs was recruited from 37 surgeries within Southampton City Primary Care Trust. Pre-piloted, closed and open-response e-questionnaires were administered to GPs asking questions on demographics, epidemiology, knowledge and management of IBD. Univariate and bivariate descriptive analyses with $90 \%$ confidence intervals were utilised. Conventional content analysis was applied to open question responses.

Results Cumulative questionnaire response rate was 50\% ( $\mathrm{n}=$ 82/164); 58\% of GPs were male, with 19 mean years (SD 9.10) practicing medicine and 13 (SD 9.41) as a GP. Estimated IBD prevalence was 471:100,000. General Practitioners consulted with 2.8 adult patients $(0.7 \%$ /total patients) with $\mathrm{IBD} /$ month and $59 \%$ independently managed those with established IBD. Short consultation times, insufficient knowledge and confidence in managing IBD and inadequate finances were identified as detrimental to GPs independently managing this patient group. Shared-care with hospital IBD services was preferred (82\%).

Conclusion A high proportion of adults with stable IBD are being managed solely by GPs. General Practitioners' lack of knowledge, confidence and resources in caring for patients with IBD inevitably occurs when managing an infrequently seen chronic condition; raising clinical governance concerns. Low exposure to this patient group questions cost-effectiveness of measures to improve GPs' knowledge-base. Findings support a shared-care approach between primary and secondary care; meeting the long-term health needs of adults with IBD.

\section{REFERENCES}

1 Rubin GP, Hungin AP, Kelly PJ, Ling J. Inflammatory bowel disease: epidemiology and management in an English general practice population. Alim Pharmacol Ther 2000;14(12):1553-59

2 Stone MA, Mayberry JF, Baker R. Prevalence and management of inflammatory bowel disease: a cross-sectional study from central England. Eur I Gastroenterol Hepatol. 2003;15(12):1275-80

3 The IBD Standards Group. Standards for the healthcare of people who have Inflammatory Bowel Disease (IBD). IBD Standards. 2013 Update. Brighton: Oyster Healthcare Communications Ltd 2013

Disclosure of Interest None Declared.

\section{PWE-109 A METABOLOMIC PROFILING STUDY OF A CHEMICALLY-INDUCED MOUSE MODEL OF INTESTINAL INFLAMMATION}

S Reade*, CA Duckworth, T Khalid, A Mayor, R Aggio, DM Pritchard, CS Probert. Gastroenterology, University of Liverpool, Liverpool, UK

\subsection{6/gutjnl-2014-307263.369}

Introduction A non-invasive, IBD-specific biomarker would be clinically useful. We have reported the changes in volatile organic metabolites (VOM) in human IBD. Human studies are limited by the variation in diet and the unpredictability of the disease. Animal models have been established to study many aspects of IBD. We report the first study of VOMs in murine DSS-colitis.

Methods C57BL/6 female mice at 9-10 weeks old were administered $4.25 \%$ dextran sulphate sodium (DSS) in their drinking water for 5 days in order to induce colitis. Clinical parameters of body weight loss, stool consistency and presence of rectal bleeding were assessed daily. Mice were culled at days 0 ( $\mathrm{n}=$ 11), $5(\mathrm{n}=11), 8(\mathrm{n}=11)$ and $11(\mathrm{n}=8)$; colonic, caecal, small bowel contents, mid-large bowel and distal small bowel tissue were taken. VOM profiles for each were analysed using SPME with a CAR/PDMS/DVB fibre and gas chromatographymass spectrometry. Histology of the distal colon confirmed the presence of colitis; this was graded from none to mild or moderate/severe.

Results Histology confirmed the presence of colitis. VOM profiles for each sample at days 5,8 and 11 were compared with day 0 . The presence/absence data were used as independent variables in a chi-squared statistical test. In the colonic content, 106 compounds were identified across all groups; 13, 22 and 10 significantly varied with presence/absence between day 0 and day 5,8 and 11, respectively. A t-test was performed on the abundance of compounds present in at least $60 \%$ of samples in one condition. A total of 29 compounds were identified; 9, 6 and 7 VOCs were present at significantly different levels between day 0 and day 5, 8 and 11, respectively. Significance levels for both chi-squared and t-tests were set at $\mathrm{p}<0.05$ and a fold difference of ${ }^{32}$. Principal component analysis (PCA) of the raw data showed clear separation between the different stages of the disease. A PCA biplot revealed that butanal, propanal, methyl propionate, ethyl acetate, ethyl propionate and 2,3-butanedione were responsible for the main separation between day 0 and day $5 / 8$ of colitis.

Conclusion Typical clinical and histological features of colitis commenced on day 5, were maximal at day 8 and mice showed signs of recovery between days 8 and 11 . The VOM results reflect this timescale, suggesting that metabolic disease profiling is able to represent the different stages of colitis. Further investigation of these differences could deepen our understanding of the pathogenesis of IBD.

Disclosure of Interest None Declared.

\section{PWE-110 A ROLE FOR PEROXIREDOXIN-4 IN A MURINE COLITIS MODEL OF INTESTINAL INFLAMMATION}

${ }^{1} \mathrm{~S}$ Pfeuffer*, ${ }^{1} \mathrm{~N}$ Trost, ${ }^{1} \mathrm{~K}$ Aden, ${ }^{1,2} \mathrm{~S}$ Schreiber, ${ }^{1} \mathrm{~S}$ Lipinski, ${ }^{1} \mathrm{P}$ Rosenstiel. ${ }^{1}$ Institute of Clinical Molecular Biology, Christian-Albrechts-University Kiel, ${ }^{2}$ First Medical Department, University Hospital Schleswig-Holstein, Kiel, Germany

\subsection{6/gutjnl-2014-307263.370}

Introduction Peroxiredoxins are a family of highly conserved antioxidant proteins. Beside its role in the reduction of peroxides, Peroxiredoxin-4 $(\operatorname{Prdx} 4)$ has been shown to play a role in the modulation of pro-inflammatory signalling cascades. Our group has previously demonstrated that $\operatorname{Prdx} 4$ is expressed in the intestinal mucosa and upregulated upon stimulation with the bacterial cell wall component muramyl-dipeptide (MDP). In addition, siRNA-mediated downregulation of $\operatorname{Prdx} 4$ increased MDP-induced NF- $\kappa B$ signalling. We therefore generated a murine Prdx4-knockout model to address the relevance of $\operatorname{Prdx} 4$ in the intestinal immune response in vivo.

Methods In this study, two different Prdx4-knockout mouse lines were used: A constitutive $\operatorname{Prdx} 4^{/-}$knockout strain, in which global Prdx4 expression was deleted and a conditional mouse line that specifically lacked $\operatorname{Prdx} 4$ in the intestinal epithelium $\left(\operatorname{Prd} x 4^{\triangle \mathrm{IEC} / \Delta \mathrm{IEC}}\right)$. Intestinal inflammation was induced by administration of dextran-sodium-sulfate (DSS) in the drinking water of $\operatorname{Prd} x 4^{-/-}, \operatorname{Prdx} 4^{\Delta \mathrm{IEC} / \Delta \mathrm{IEC}}$ and respective littermate control 